\begin{tabular}{|c|l|}
\hline Title & High mobility of Iattice molecules and defects during the early stage of protein crystall lization \\
\hline Author(s) & Yamazaki, Tomoya; V an Driessche, A lexander E. S.; Kimura, Y uki \\
\hline Citation & $\begin{array}{l}\text { Soft matter, 16(8), 1955-1960 } \\
\text { https://doi.org/10.1039/c9sm02382h }\end{array}$ \\
\hline Issue Date & 2020-02-28 \\
\hline Doc URL & http://hdl.handle.net/2115/80511 \\
\hline Type & article(author version) \\
\hline File Information & softmatter_16(8)_1955_1960.pdf \\
\hline
\end{tabular}

Instructions for use 


\title{
High mobility of lattice molecules and defects during the early stage of protein crystallization
}

\author{
Tomoya Yamazaki, ${ }^{a}$ Alexander E. S. Van Driessche ${ }^{b}$ and Yuki Kimura*a \\ Protein crystals are expected to be useful not only for their molecular structure analysis but also as \\ functional materials due to their unique properties. Although the generation and the propagation of \\ defects during crystallization play critical roles in the final properties of protein crystals, the \\ dynamics of these processes are poorly understood. By time-resolved liquid-cell transmission \\ electron microscopy, we observed that nanosized crystal defects are surprisingly mobile during the \\ early stages of the crystallization of a lysozyme as a model protein. This highly dynamic behavior of \\ defects reveals that the lattice molecules are mobile throughout the crystal structure. Moreover, the \\ disappearance of the defects indicated that intermolecular bonds can break and reform rapidly with \\ little energetic cost, as reported in theoretical studies. All these findings are in marked contrast to the \\ generally accepted notion that crystal lattices are rigid with very limited mobility of individual lattice \\ molecules.
}

\section{Introduction}

The presence of defects in crystals plays a crucial role in their overall quality, ${ }^{1}$ physical properties, ${ }^{2}$ and growth $^{3,4} /$ dissolution $^{5}$ behaviour. Through in situ observations at the nanoscale, our comprehension of the dynamics of defects in hard crystalline materials, such as metals, has progressed remarkably in recent decades. ${ }^{6-8}$ However, our understanding of defect behaviour in crystals of soft matter remains limited as a result of technical limitations. Protein crystals have characteristic features of soft matter, ${ }^{9}$ such as high molecular weights and low elastic constants ${ }^{10}$. Protein crystals, especially large defect-free ones, ${ }^{11}$ are in high demand for the high-resolution analysis of the molecular structures of these proteins. Consequently, the quality of protein crystals has been extensively evaluated by visualization of defects under static or dynamic conditions by using several techniques such as X-ray topography, ${ }^{12,13}$ laser scattering tomography, ${ }^{14}$ optical microscopy, ${ }^{1,5}$ atomic force microscopy (AFM), ${ }^{12,15}$ or electron microscopy. ${ }^{16-18} \mathrm{X}$-ray and electron beam-based methods are useful for evaluating crystals that have finished growing, whereas microscopy methods are useful for studying the origin of defects during the crystallization process. In particular, AFM is a powerful tool for observing the dynamics of defects on the surfaces of protein crystal with molecular resolution. Various types of defects, such as point defects, dislocation outcrops, and stacking faults, have been characterized, and it has been shown that the incorporation of impurities or microcrystals can act as a trigger for defect formation. ${ }^{12}$ However,

\footnotetext{
a. Institute of Low Temperature Science, Hokkaido University, Kita-19, Nishi-8, Kita-ku, Sapporo, 060-0819, Japan. E-mail:

ykimura@lowtem.hokudai.ac.jp

b.Université Grenoble Alpes, Université Savoie Mont Blanc, CNRS, IRD, IFSTTAR, ISTerre, F-38000, Grenoble, France.
}

AFM can only provide information about the solidliquid interface; moreover, the solution conditions that are suitable for AFM imaging are always relatively close to the thermodynamically equilibrium (compared with the typical high supersaturation conditions that prevail energetic barriers during the processes of nucleation and initial crystal growth). Consequently, the behaviour of defects inside crystals during the early stages of crystallization remains unknown. To examine the dynamics of defects inside protein crystals, we therefore require observations that are simultaneously three-dimensional, time-resolved, and performed at the nanoscale.

The specific lattice properties of a given crystalline material play a key role in the dynamics of its defects. In the case of protein crystals, lattice molecules can undergo rather rapid rocking motions, ${ }^{19}$ and the molecular contacts are thought to be dynamic. ${ }^{20}$ Unlike most inorganic solids, protein crystals also show a remarkable ability to incorporate a wide variety of relatively large particles, such as homologous microcrystals, ${ }^{21}$ foreign nanocrystals, ${ }^{22}$ or polymer fibres, ${ }^{23}$ without significant disturbance the long-range order of the lattice periodicity of the crystals. This accommodating capacity of protein crystals has been attributed to the prevailing noncovalent water-mediated lattice contacts. ${ }^{20}$ It has recently been demonstrated that the adaptive properties of macromolecular lattices can be further improved when the lattices are integrated into a hydrogel network that permits the protein crystals to expand isotopically and to return to their original volume while retaining their periodic order. ${ }^{24}$ However, in a quite contradictory manner, perfectly shaped macroscopic protein crystals often display poor diffraction properties. To shed light on the origin of these remarkable and apparently incompatible lattice properties, we used in situ 
liquid-cell transmission electron microscopy (LCTEM $)^{25}$ to observe the dynamics of defects inside crystals of lysozyme protein at the nanoscale. Our observations provide direct evidence of the true nature of the macromolecular crystalline lattice, which is much more flexible and more dynamic than previously assumed, and expand on current knowledge of the potential use of protein crystals as functional materials, as has been recently reported. ${ }^{26-}$ 28

\section{Results and Discussion}

During the in situ LC-TEM imaging, we observed the dynamics of defect inside orthorhombic and tetragonal crystals of lysozyme, which can be observed under our experimental conditions (see Experimental section). ${ }^{29}$ An elongated orthorhombic crystal and a pseudo-hexagonal-shaped tetragonal crystal are shown in the same field of view in Fig. 1A. Both polymorphs displayed two types of characteristic TEM contrast originating from defects present inside the crystals: (1) bright areas associated with mass-volume contrast originating from voids, and (2) paired areas of bright and dark contrast associated with the diffracted electrons that arise from (slightly) misaligned crystalline blocks (mosaicity). In principle, the former group of contrasts arises from incoherent elastic scattering (Rutherford scattering) of electrons, and they depend on the atomic number, density, and volume of the specimen. ${ }^{30}$ For the Case 2, the contrast is linked to coherent elastic scattering, ${ }^{30}$ and electrons diffracted by the crystal lattice planes, which produce bright and dark areas on the TEM image.

\section{TEM Contrast of a Void and its Movements.}

Rounded bright areas (see Fig. 1) with maximum sizes of $\sim 60$ and $\sim 200 \mathrm{~nm}$ were observed in tetragonal (Fig. S1) and orthorhombic lysozyme crystals (Figs. 2A and S2), respectively. As these bright areas were not coupled to dark areas in the crystals, they were the result of differences in the absolute crystal mass-volume ${ }^{30}$ in other words, the scattering factor for electrons in this area was lower than that in the surrounding area of the specimen. Therefore these contrasts arose either from voids, which form due to the aggregation of vacancies inside the crystals, ${ }^{31}$ or from etch pits located at the crystal surface. ${ }^{32}$ However, the latter case is unlikely because etch pits form only in undersaturated solutions (the driving force of the etch pit formation is the difference in the chemical potential between the solid and liquid phase), whereas these bright areas were always observed in growing crystals. Instead, the decisive factor in void formation is the concentration of vacancies in the crystal; ${ }^{31}$ if the

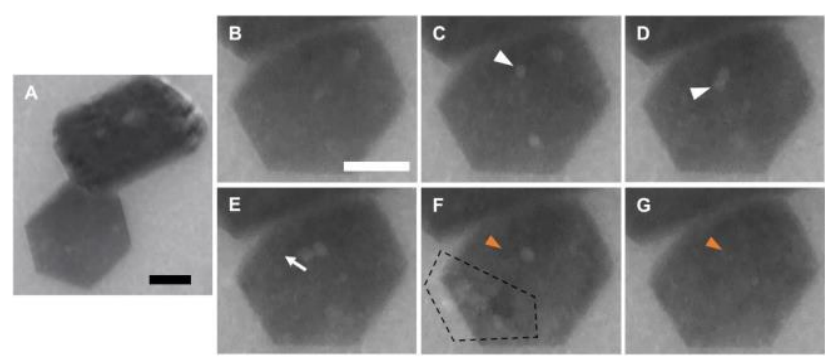

Figure 1. TEM contrast behaviors in a tetragonal lysozyme crystal. (A) Orthorhombic (upper) and tetragonal (lower) lysozyme crystals in the same field of view. (B-G) Enlarged images of the tetragonal lysozyme crystal shown in (A). Images B to $G$ were captured 217 (B), 229 (C), 239 (D), 251 $(E), 261(F)$, and $282 \mathrm{~s}(\mathrm{G})$ after the crystal was irradiated by the electron beam. The white and red arrowheads indicate locations of the formation and disappearance of voids, respectively. The white arrow indicates the direction of movement of the void. The region enclosed by the broken line contains paired areas of bright and dark contrasts. The scale bars are $200 \mathrm{~nm}$, and the scale in $(C-G)$ is the same as that in $(B)$.

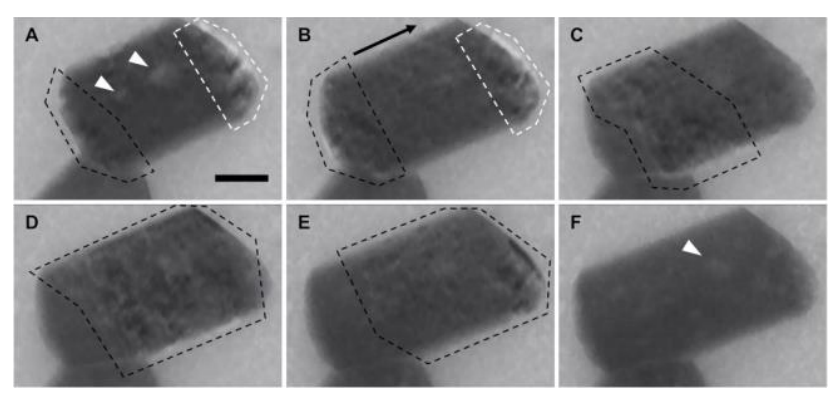

Figure 2. TEM contrast behaviors in an orthorhombic lysozyme crystal. (A-F) Enlarged images of the orthorhombic lysozyme crystal shown in Fig. 1A. Images AF were captured 355 (A), 395 (B), 415 (C), 425 (D), 435 (E), and $445 \mathrm{~s}(\mathrm{~F})$ after the crystal was irradiated by the electron beam. The white arrowheads indicate the locations of voids. The regions enclosed by the white and black broken lines show paired areas of bright and dark contrast that maintain their position or move, respectively. The scale bar is $200 \mathrm{~nm}$, and the scale in $(B-F)$ is the same as that in $(A)$.

number of vacancies increases beyond the equilibrium concentration, they begin to form voids to reduce their total energy. Furthermore, the shape of the etch pits on the (110) face of tetragonal crystals is boat-like and elongated in the $\langle 110\rangle$ direction $^{33}$ (even if the size of an etch pit is of a submicron order $\left.{ }^{34}\right)$. Consequently, these bright areas do not represent etch pits but, instead, correspond to voids within the crystal. This type of large void has not been reported before. In principle, the shape of the voids generally appears to be spherical. However, we also observed those that were elongated (Fig. S3). 
The elongated voids inside the orthorhombic crystals was over $100 \mathrm{~nm}$ in length. The length of these voids was not parallel to the $c$-axis of the crystal. The $c$ axis should be the thermodynamically-favored direction of the voids due to the shape of the crystal. These non-parallel voids imply that the free energy of the system is larger than those with voids along the $c$-axis, if the shapes are the same, suggesting that these voids are not thermodynamically favored. The formation, mobility, and disappearance of voids was observed in tetragonal and orthorhombic crystals with sizes of $<500 \mathrm{~nm}$ and $<1200 \mathrm{~nm}$ along the $c$-axis directions, respectively (Figs. 1, 2, S1, and S2 and Movies S1 and S2).

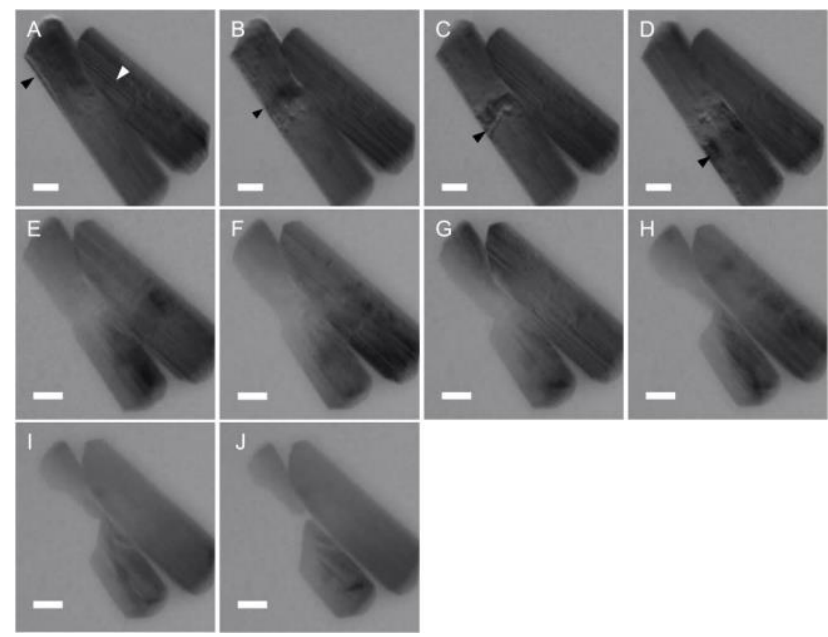

Figure 3. TEM contrast behaviours in dissolving orthorhombic lysozyme crystals. Some dark band contrast (indicated by black arrowheads) appeared at the center of the left-hand crystal (A-D). From these points of the contrast, the crystal started to dissolve, indicating that these contrasts originate from screw dislocations and that each dissolved part corresponds to the formation of an etch pit, as strain fields around screw dislocations facilitate the dissolution of the crystal $(\mathrm{E}-\mathrm{J})$. The white arrowhead indicates a contrast arising from a planar defect. Images B$\mathrm{J}$ were captured 20 (B), 30 (C), 50 (D), 225 (E), $245(\mathrm{~F}), 255$ (G), $275(\mathrm{H}), 285(\mathrm{I})$, and $295 \mathrm{~s}(\mathrm{~J})$ after Image A (the crystals began to be irradiated by the electron beam approximately $460 \mathrm{~s}$ previously). The scale bar is $200 \mathrm{~nm}$, and the scale is the same in all images.

By tracking the movement of voids shown in Fig. 1, we estimated their diffusivity to be $(3-7) \times$ $10^{-17} \mathrm{~m}^{2} \cdot \mathrm{s}^{-1}$. Because a void is usually formed through aggregation of supersaturated lattice vacancies $^{31}$, the movements of these voids demonstrate that lattice vacancies are mobile. This means that lysozyme molecules in the crystal are also mobile because the movement of vacancies has to be accompanied by that of lattice molecules. This type of mobility has been previously observed in metal nanoparticles and is promoted by the increased diffusion coefficient of the lattice molecules due to lattice softening, a size-dependent effect of nanoparticles ${ }^{35}$. This increased lattice mobility is exemplified by the immediate mixing of $\sim 4 \mathrm{~nm}$ gold nanoparticle with copper at room temperature to form a metal alloy ${ }^{35}$. If we assume that a gold nanoparticle is spherical, then the number of gold atoms in such a nanoparticle is approximately $2 \times 10^{3}$. If we now assume that a spherical crystal of tetragonal lysozyme has a diameter of $400 \mathrm{~nm}$ (approximately equal to the size of the crystals that we observed), the number of protein molecules in the crystal exceeds $10^{6}$, which is approximately three orders of magnitude larger than that of a gold nanoparticle that shows lattice softening. Therefore, the movement of voids inside lysozyme crystals suggests that lattice softening can occur in protein crystals that are much larger than in the corresponding case for metal nanocrystals. This remarkable behavior might well be the key to explaining why both the positive and negative lattice properties observed for protein crystals such as selfhealing ${ }^{24}$ and poor diffraction properties of apparently perfect crystals: the high mobility of lattice molecules would permit the crystals to expand and to return to their original state with relatively ease while, simultaneously, the high mobility of the molecules results in deterioration of the quality of the diffraction spots.

\section{The Effect of Electron Beams on Defect Formation in Crystals.}

The electron fluxes in this study were between $3.2 \times 10^{2}$ and $2.9 \times 10^{3} \mathrm{e} \cdot \mathrm{nm}^{-2} \cdot \mathrm{s}^{-1}$. When flux is below the former value, lysozyme crystal growth is not significantly influenced by the electron beam ${ }^{29}$. However, when the flux is above the latter value, the lysozyme crystals are eventually dissolved or destroyed $^{29}$. This outcome is a direct consequence of the interactions of the electron beam with the crystal (e.g., the ionization of bonds resulting in bond breaking and the formation of defects) and the growth solution (e.g., radiolysis of water and other chemical species). Therefore, the interactions with crystals may influence the formation and/or dynamics of the voids. Cryo-TEM observations revealed that there is a critical value of total accumulated dose $\left(9 \times 10^{2} \mathrm{e} \cdot \mathrm{nm}^{-2}\right)$ that deteriorates the quality of the diffraction spots ${ }^{36}$. Therefore, a lysozyme crystal under static conditions will experience beam damage once the total accumulated dose is reached. Therefore, the number of defects, such as voids, should increase when the total accumulated dose is surpassed. To verify this mechanism, we measured the number and total area 
of voids in lysozyme crystals exposed to an electron beam with an electron flux between $3.2 \times 10^{2}$ and $2.9 \times 10^{3} \mathrm{e} \cdot \mathrm{nm}^{-2} \cdot \mathrm{s}^{-1}$ (Figs. S4-S6). We observed crystals where the movement of voids was visible (Fig. 1) and measured the number and total area of the voids during $249 \mathrm{~s}$. The accumulated electron dose the crystals received was between $8.0 \times 10^{4}$ $7.2 \times 10^{5} \mathrm{e} \cdot \mathrm{nm}^{-2}$ (Fig. S4). Neither number nor area significantly increased during observations. The total area of voids in the projection constituted 4-6\% of the crystal (Fig. S4). Under similar conditions to those of Fig. S4, we did not observe any increase in the number and area of voids. However, the total area of the voids in the projection was slightly lower, i.e., 1-2\%, compared to that of the other crystal (Fig. S5). In addition, a crystal that did not show voids at the start of the observations did not develop any voids during irradiation (Fig. S6). In summary, we did not observe any significant effects of the beams on the formation of voids under experimental conditions. These results confirm that the observed behavior of defects is intrinsic to the crystals, rather than being beam-induced effects.

\section{TEM Contrast of a Crystal Block and its Movements.}

The paired areas of bright and dark contrasts visible in Figs. 1F and 2A-E arise from electron diffraction by lattice planes with different orientations. Thus, each contrast is associated with a single crystal block that has a slightly different crystallographic orientation to that of the surrounding blocks. Such contrasts can appear if the crystalline block contains at least four to five molecular layers, ${ }^{37}$ corresponding to a $\sim 10 \mathrm{~nm}$ thickness inside the protein crystal. These contrasts are usually generated at one edge of a crystal and move to the opposite edge. They crossed the entire crystal along the $\langle 001\rangle$ direction (Fig. S2 and Movie S2) with a maximum propagation rate of $\sim 50 \mathrm{~nm} \cdot \mathrm{s}^{-1}$. These events were repeatedly observed in the same crystal.

Step movement on the crystal surface, and the accompanying changes in crystal thickness, cannot have a significant effect on the paired areas of bright and dark contrast. This is because the typical shape of elementary and macro-step patterns on the crystal surface of orthorhombic ${ }^{38}$ and tetragonal crystals $^{39,40}$ do not correspond to those of the areas of the contrast observed by LC-TEM. It is also difficult to envisage that a crystal block can diffuse through the bulk of a crystal lattice. Therefore, only two plausible options remain to explain the rapid movement of these contrast pairs: (I) the crystal blocks are continuously generated and disappeared by the breaking and recombination of bonds, or (II) they are always present in the crystal but the

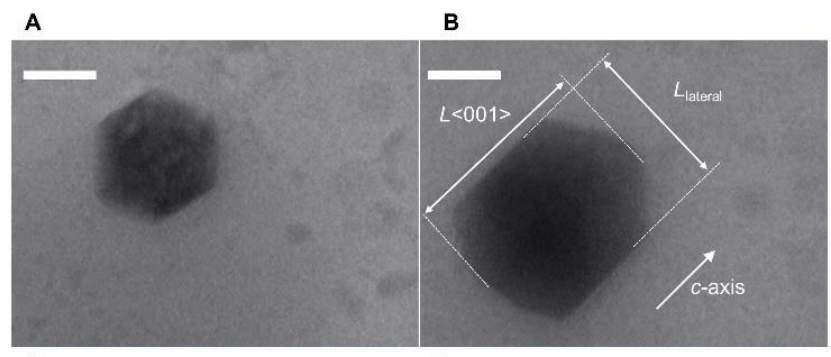

C

D
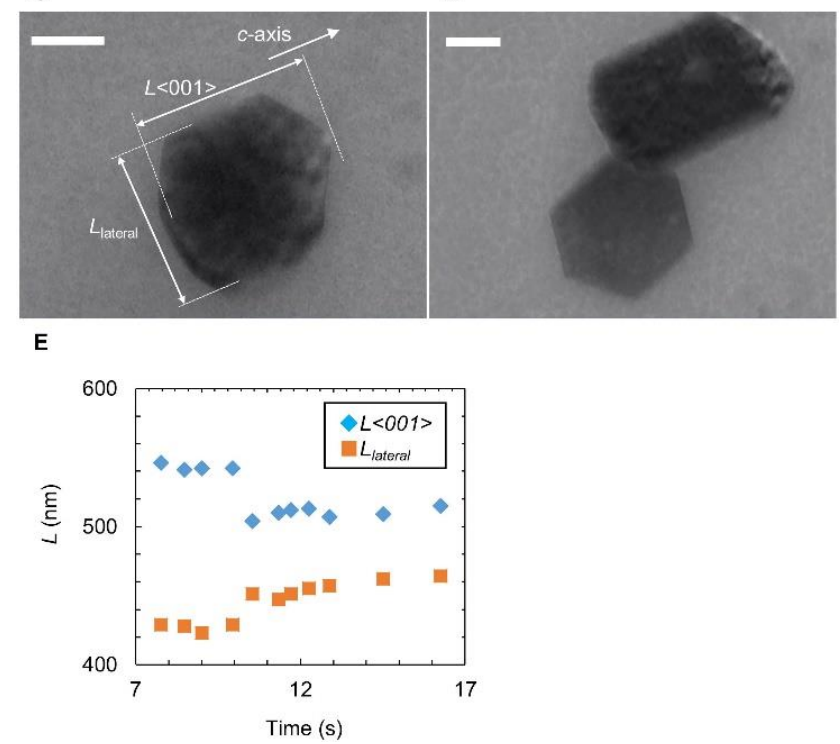

Figure 4. Possible phase transition of a lysozyme crystal from tetragonal to orthorhombic. (A-B) Growing pseudohexagonal crystal (tetragonal). (C) The $c$-axis direction of the crystal in (B) was suddenly inclined by $20^{\circ}$. After this event, the crystal grew into the upper crystal in (D). At about this time, $\mathrm{L}<001>$ was reduced by $\sim 40 \mathrm{~nm}$ and expanded laterally by $\sim 20 \mathrm{~nm}$ within $2.3 \mathrm{~s}(\mathrm{E})$. Images $\mathrm{B}-\mathrm{D}$ were captured 181 (B), 184 (C), and $290 \mathrm{~s}$ (D) after Image A. The scale bars are $200 \mathrm{~nm}$.

contrasts arising from their presence only appears under certain diffraction conditions. For the latter case, a slightly bent crystal would show a band contrast if specific crystal planes satisfy the Bragg condition, ${ }^{30}$ i.e., the so-called bend contour. Such a band contrast would move when the crystallographic orientation changes slightly with respect to the beam axis. One of our observations showed that a band contrast can highlight the specific contrasts of planar defects and dislocations in lysozyme crystals (Fig. 3 and Movies S3 and S4). Thus, bend contours highlight defects because those parts of a crystal plane in which defects exist do not satisfy the Bragg condition and perturb the band contrast. This suggests that paired areas of bright and dark contrast arising from different crystal blocks would also be highlighted. Additional observations showed the existence of paired areas at the edge of an orthorhombic crystal that stayed in the same region. 
This finding indicates that a single band contrast (where the Bragg condition does not change) should highlight these structures; however, the contrasts frequently changed (Figs. 2 and S7 and Movie S2). Therefore, the boundaries of the crystal blocks were in continuous motion. Moreover, the block structure, which spread across an entire crystal, disappeared as the crystal grew, while the band contrast remained detectable, indicating that the crystal blocks merged and created a crystal with a single-crystalline domain (Fig. S8). On the basis of the above observations, we conclude that crystal blocks form and disappear continuously. For crystal blocks to (re)align, molecules contained in the grain boundaries need to be mobile and to undergo rapid bond breaking and recombination. This again highlights the highly dynamic character of lattice molecules inside the crystal. Although the experimental conditions are completely different, this realignment of lattice molecules and crystal blocks might be related to the improvement in the quality of diffraction patterns observed for protein crystals after annealing during cryocrystallography. ${ }^{41,42}$

\section{Flexibility of Lattice Induced Solid-Solid Phase Transition.}

Under the experimental conditions used during our LC-TEM experiments, orthorhombic crystals are more stable than tetragonal crystal (see Experimental section). By taking into account the dynamic properties of the lysozyme lattice molecules discussed so far, it should be feasible for solid-solid phase transitions to occur at a reduced thermodynamic cost. Indeed, we observed that crystals suddenly transformed from one polymorph to the other, i.e. tetragonal lysozyme crystals (the less-stable polymorph), which have a typical pseudohexagonal morphology, transformed into the elongated crystals associated with the orthorhombic lysozyme polymorph (Fig. S7). This transformation from tetragonal to orthorhombic lysozyme crystals occurred within a few seconds (Fig. 4 and Movie S5). During this phase transition, no dissolution step was involved, indicating that the polymorph transformation was solid-state mediated. This phenomenon can only be explained if the molecules inside the lattice are highly dynamic and readily change their orientation by breaking and forming new bonds. This property of the crystalline lattice permits and/or promotes a solid-solid phase transition from a metastable to a stable crystalline phase under near-equilibrium condition for both polymorphs. In addition, the transition may induce many vacancies in the crystal. We observed the void where the elongated direction was not parallel to the c-axis of the orthorhombic crystal (Fig. S3). The formation of such voids may be related to the highly supersaturated vacancy conditions (i.e. many vacancies in a crystal) because a driving force is required for the void to exist in the thermodynamically unfavored direction.

\section{Conclusions}

Our in situ LC-TEM observations revealed that lattice molecules of protein crystals are highly mobile, in stark contrast to the textbook view of rigid lattice properties of inorganic crystalline materials. We also provide evidence that, besides the mobility of the lattice molecules, the crystal bonds can be rapidly broken and re-established. Our experimental observations are consistent with a recently proposed hypothesis that intermolecular contacts in protein crystals are dynamic. ${ }^{20}$ This highly dynamic character of lattice molecules and their bonding provides a rationale to explain the extraordinary expansion, contraction, and self-healing capacity of ferritin-hydrogel composite crystals, ${ }^{24}$ and the remarkable ability of protein crystals to incorporate homologous or heterologous particles without perturbation of the long-range order of their lattice periodicity. These characteristic features of protein crystals indicate the possibility of developing a new class of functional materials that are inaccessible via purely inorganic materials and provide a basis for exploring the self-healing capacity of crystalline structures. ${ }^{43}$

\section{Experimental}

\section{Materials and Solution Preparation.}

Six-times-recrystallized lysozyme powder (lot no. E40314; Seikagaku Kogyo, Tokyo) was used without further purification. Solid $\mathrm{NaCl}$ (99.99\%; Wako Pure Chemical Industries, Ltd., Osaka) was used as a precipitant to crystallize the lysozyme. Stock solutions of both materials were prepared by dissolving the appropriate amounts in $50 \mathrm{mM}$ aqueous sodium acetate solution $(\mathrm{pH} 4.5)$. The concentration of the lysozyme stock solutions was measured by means of UV absorption spectrometry (SmartSpec Plus; Bio-Rad Laboratories, Hercules, CA). The crystallization solution of the lysozyme was prepared by mixing appropriate amounts of a stock solution and the buffer solution.

\section{Transmission Electron Microscopy and the Liquid Cell.}

We used a Hitachi H-8100 transmission electron microscope with a $\mathrm{LaB}_{6}$ filament operating at an acceleration voltage of $200 \mathrm{kV}$. Images and movies were recorded by using an XR-611 TEM camera 
(Advanced Microscopy Techniques Corp., Woburn, MA) with a frame interval of $157 \mathrm{~ms}$. The electron flux was measured by using a probe-current detector combined with a Model 6485 picoammeter (Keithley Instruments, Solon $\mathrm{OH}$ ) installed in the $\mathrm{H}-8100$ microscope and it was controlled to be less than $2.9 \times 10^{3}$ electron $\mathrm{nm}^{-2} \cdot \mathrm{s}^{-1}$, as above this value, destruction of the lysozyme crystal began to occur in the crystallization solution ${ }^{29}$. A Poseidon TEM holder (Protochips Inc., Morrisville, NC) with a liquid cell was used for imaging of the liquid samples (Fig. S9). The liquid cell consisted of a pair of Echips (which are silicon plates with an electrontransparent window made of an amorphous silicon nitride membrane), and two Viton O-rings. The Echips were separated by $500-\mathrm{nm}$-thick spacers to create a thin liquid layer that acted as a flow path for the sample solution. The thickness of the observed solution layer was greater than that of the spacer, but was less than $1 \mu \mathrm{m}$, because of the expansion resulting from the vacuum in the electron microscope ${ }^{44}$. Therefore, the observed crystals were thinner than $1 \mu \mathrm{m}$, although their exact thicknesses were unknown. Before the assembly of the liquid cell, the E-chips were hydrophilized by plasma ion bombardment.

\section{Time-Resolved LC-TEM Observations.}

We used two crystallization solutions with different lysozyme concentrations. The crystallization solution with the higher concentration [crystallization solution (H), in Fig. S9] was used initially to fill the liquid cell before the observations. This solution was prepared by mixing the lysozyme solution with the $\mathrm{NaCl}$ solution immediately before filling the liquid cell. The resulting solution contained $50 \mathrm{mg} \cdot \mathrm{mL}^{-1}$ lysozyme and $50 \mathrm{mg} \cdot \mathrm{mL}^{-1}$ $\mathrm{NaCl}$ and was not filtered. The crystallization solution with the lower lysozyme concentration [crystallization solution (L), in Fig. S9] contained 15 $\mathrm{mg} \cdot \mathrm{mL}^{-1}$ lysozyme and $50 \mathrm{mg} \cdot \mathrm{mL}^{-1} \mathrm{NaCl}$, and this solution was flowed through the liquid cell during the TEM observations. This solution was filtered through a cellulose acetate filter with $0.20 \mu \mathrm{m}$ pores. During the observations, the solution was passed continuously through the liquid cell at a rate of 1-3 $\mu \mathrm{L} \cdot \mathrm{min}^{-1}$ by using a syringe pump. All the experiments were performed at $24{ }^{\circ} \mathrm{C}$. Under these conditions, orthorhombic and tetragonal lysozyme crystal form in the crystallization solution enclosed in the liquid cell..$^{29}$ The solubility of the orthorhombic lysozyme crystal in the crystallization solution at $24{ }^{\circ} \mathrm{C}$ is slightly lower than that of tetragonal lysozyme crystal (i.e., the solubilities of the orthorhombic and tetragonal phases were 3.19 and $3.30 \mathrm{mg} \cdot \mathrm{mL}^{-1}$, respectively $\left.{ }^{45,46}\right)$. Therefore, the orthorhombic lysozyme crystal is more stable than the tetragonal lysozyme crystal under our experimental condition.

\section{Measurements of the Total Area and Number of Voids in the Crystals.}

We processed the TEM images using Image $^{47}$ to measure the total area and number of voids in the crystals. After converting the images into 8-bit, we selected the crystalline areas that the voids were observed in using "polygon selections." Then, we processed the images using the "subtract background" function with a rolling ball radius of 10 pixels and by checking the e to create a flat background. After enhancing the contrast, the images were filtered using "Gaussian blur (the value of sigma is 1 or 2)". We selected the threshold value by comparing the size of voids in the processed and original images to convert them into binary images. Next, we applied "analyze particles (size: 10 to infinity)" function, to obtain the area and number of voids. Due to this process, we were unable to analyze voids with a diameter of less than 10 pixels, which corresponds to 24 or $32 \mathrm{~nm}$ in Figs. S4 and S5, respectively.

\section{Conflicts of interest}

There are no conflicts to declare.

\section{Acknowledgements}

We thank K. Tsukamoto and S. Ishizuka for their suggestions and encouragement, and F. Erika for assistance with the TEM observations. This work was supported by JSPS KAKENHI Grant Numbers JP15H05731, JP18J01470, and JP18K14134. TY is grateful for the Research Fellowship of the Japan Society for the Promotion of Science for Young Scientists.

\section{Notes and references}

1 I. Yoshizaki, S. Fukuyama, H. Koizumi, M. Tachibana, K. Kojima, Y. Matsuura, M. Tanaka, N. Igarashi, A. Kadowaki, L. Rong, S. Adachi, S. Yoda and H. Komatsu, J. Cryst. Growth, 2006, 290, 185191.

2 N. Zhang, P. Carrez and R. Shahsavari, ACS Appl. Mater. Interfaces, 2017, 9, 1496-1506.

3 Y. Tominaga, M. Maruyama, M. Yoshimura, H. Koizumi, M. Tachibana, S. Sugiyama, H. Adachi, K. Tsukamoto, H. Matsumura, K. Takano, S.

Murakami, T. Inoue, H. Y. Yoshikawa and Y. Mori, Nat. Photonics, 2016, 10, 723-726.

4 K. Tsukamoto, Prog. Cryst. Growth Charact. Mater., 2016, 62, 111-125. 
5 G. Sazaki, K. Tsukamoto, S. Yai, M. Okada and K. Nakajima, Cryst. Growth Des., 2005, 5, 1729-1735.

6 S. H. Oh, M. Legros, D. Kiener and G. Dehm, Nat. Mater., 2009, 8, 95-100.

7 W. Xu, Y. Zhang, G. Cheng, W. Jian, P. C. Millett, C. C. Koch, S. N. Mathaudhu and Y. Zhu, Nat. Commun., 2013, 4, 2288.

8 L. Luo, M. Su, P. Yan, L. Zou, D. K. Schreiber, D. R. Baer, Z. Zhu, G. Zhou, Y. Wang, S. M. Bruemmer, Z. Xu and C. Wang, Nat. Mater., 2018, 17, 514-518.

9 D. Fusco and P. Charbonneau, Colloids Surfaces B Biointerfaces, 2016, 137, 22-31.

10 A. A. Chernov, J. Struct. Biol., 2003, 142, 3-21.

11 S. X. Lin, A. McPherson and R. Giegé, Cryst. Growth Des., 2007, 7, 2124-2125.

12 A. J. Malkin and R. E. Thorne, Methods, 2004, 34, 273-299.

13 H. Koizumi, M. Shimizu, M. Tachibana and K. Kojima, Phys. Status Solidi Appl. Mater. Sci., 2007, 204, 2688-2693.

14 K. Sato, Y. Fukuba, T. Mitsuda, K. Hirai and K. Moriya, J. Cryst. Growth, 1992, 122, 87-94.

15 S. T. Yau, B. R. Thomas, O. Galkin, O. Gliko and P. G. Vekilov, Proteins Struct. Funct. Genet., 2001, 43, 343-352.

16 H. P. Stevenson, G. Lin, C. O. Barnes, I.

Sutkeviciute, T. Krzysiak, S. C. Weiss, S. Reynolds, Y. Wu, V. Nagarajan, A. M. Makhov, R. Lawrence, E. Lamm, L. Clark, T. J. Gardella, B. G. Hogue, C. M. Ogata, J. Ahn, A. M. Gronenborn, J. F. Conway, J. P. Vilardaga, A. E. Cohen and G. Calero, Acta Crystallogr. Sect. D Struct. Biol., 2016, 72, 603-615.

17 N. Braun, J. Tack, M. Fischer, A. Bacher, L.

Bachmann and S. Weinkauf, J. Cryst. Growth, 2000, 212, 270-282.

18 M. Gallagher-Jones, C. Ophus, K. C. Bustillo, D. R. Boyer, O. Panova, C. Glynn, C. Zee, J. Ciston, K. C. Mancia, A. M. Minor and J. A. Rodriguez, Commun. Biol., 2019, 2, 26.

19 P. Ma, Y. Xue, N. Coquelle, J. D. Haller, T. Yuwen, I. Ayala, O. Mikhailovskii, D. Willbold, J.-P. Colletier, N. R. Skrynnikov and P. Schanda, Nat. Commun., 2015, 6, 8361.

20 M. Dimova and Y. D. Devedjiev, IUCrJ, 2018, 5, 130-140.

21 A. J. Malkin, Y. G. Kuznetsov and A. McPherson, Surf. Sci., 1997, 393, 95-107.

22 H. Wei, Z. Wang, J. Zhang, S. House, Y. G. Gao, L. Yang, H. Robinson, L. H. Tan, H. Xing, C. Hou, I. M. Robertson, J. M. Zuo and Y. Lu, Nat. Nanotechnol., 2011, 6, 93-97.

23 J. A. Gavira and J. M. García-Ruiz, Acta Crystallogr. Sect. D Biol. Crystallogr., 2002, 58, 1653-1656.

24 L. Zhang, J. B. Bailey, R. H. Subramanian, A. Groisman and F. A. Tezcan, Nature, 2018, 557, 8691.
25 K. L. Klein, I. M. Anderson and N. de Jonge, $J$. Microsc., 2011, 242, 117-123.

26 B. Maity, K. Fukumori, S. Abe and T. Ueno, Chem. Commun., 2016, 52, 5463-5466.

27 S. Lopez, L. Rondot, C. Leprêtre, C. MarchiDelapierre, S. Ménage and C. Cavazza, J. Am. Chem. Soc., 2017, 139, 17994-18002.

28 R. Contreras - Montoya, G. Escolano, S. Roy, M. T. Lopez - Lopez, J. M. Delgado - López, J. M. Cuerva, J. J. Díaz - Mochón, N. Ashkenasy, J. A. Gavira and L. Álvarez de Cienfuegos, Adv. Funct. Mater., 2019, 29, 1807351.

29 T. Yamazaki, Y. Kimura, P. G. Vekilov, E. Furukawa, M. Shirai, H. Matsumoto, A. E. S. Van Driessche and K. Tsukamoto, Proc. Natl. Acad. Sci., 2017, 114, 2154-2159.

30 D. B. Williams and C. B. Carter, Transmission Electron Microscopy, Springer US, Boston, MA, Second edi., 2009.

31 H. Yasuda, A. Tanaka, K. Matsumoto, N. Nitta and H. Mori, Phys. Rev. Lett., 2008, 100, 105506.

32 H. Hondoh and T. Nakada, J. Cryst. Growth, 2005, 275, 1423-1429.

33 L. A. Monaco and F. Rosenberger, J. Cryst. Growth, 1993, 129, 465-484.

34 H. Hondoh and T. Nakada, Jpn. J. Appl. Phys., 2004, 43, 4529-4532.

35 H. Yasuda and H. Mori, Z. Phys. D Atoms, Mol. Clust., 1994, 31, 131-134.

36 D. Shi, B. L. Nannenga, M. G. Iadanza and T. Gonen, Elife, 2013, 2013, 1-17.

37 K. Yonekura, K. Kato, M. Ogasawara, M. Tomita and C. Toyoshima, Proc. Natl. Acad. Sci., 2015, 112, 3368-3373.

38 Y. Matsuzuki, T. Kubota, X. Y. Liu, M. Ataka and K. J. Takano, J. Cryst. Growth, 2002, 242, 199-208.

39 P. Dold, E. Ono, K. Tsukamoto and G. Sazaki, J. Cryst. Growth, 2006, 293, 102-109.

40 A. E. S. Van Driessche and M. Sleutel, Cryst. Res. Technol., 2013, 48, 919-941.

41 J. I. Yeh and W. G. J. Hol, Acta Crystallogr. Sect. D Biol. Crystallogr., 1998, 54, 479-480.

42 J. M. Harp, D. E. Timm and G. J. Bunick, Acta Crystallogr. Sect. D Biol. Crystallogr., 1998, 54, 622-628.

43 P. Commins, H. Hara and P. Naumov, Angew. Chemie - Int. Ed., 2016, 55, 13028-13032.

44 M. E. Holtz, Y. C. Yu, J. Gao, H. D. Abruna and D. A. Muller, Microsc. Microanal., 2013, 19, 10271035.

45 F. Ewing, E. Forsythe and M. Pusey, Acta Crystallogr. Sect. D Biol. Crystallogr., 1994, 50, 424-428.

46 A. E. S. Van Driessche, J. A. Gavira, L. D. Patiño Lopez and F. Otalora, J. Cryst. Growth, 2009, 311, 3479-3484. 
47 C. A. Schneider, W. S. Rasband and K. W. Eliceiri, Nat. Methods, 2012, 9, 671-675. 